\title{
Electrophilic Azides for Materials Synthesis and Chemical Biology
}

\author{
Sheng Xie, ${ }^{1 *}$ Madanodaya Sundhoro, ${ }^{2}$ K. N. Houk, ${ }^{3 *}$ Mingdi Yan $^{2 *}$ \\ ${ }^{1}$ State Key Laboratory of Chemo/Biosensing and Chemometrics, College of Chemistry and \\ Chemical Engineering, Hunan University, Changsha 410082, P. R. China \\ ${ }^{2}$ Department of Chemistry, University of Massachusetts Lowell, 1 University Ave., Lowell, MA \\ 01854, USA \\ ${ }^{3}$ Department of Chemistry and Biochemistry, University of California, Los Angeles, CA 90095
}

\section{Table of Contents}

1. Cycloaddition reaction of sulfonyl azide and phenylacetaldehyde enamine.................S1

2. Synthesis of 4-methyl- $N$-(morpholinomethylene)benzenesulfonamide....................S1

3. Figure S1. ${ }^{1} \mathrm{H}$ NMR spectrum of 4-methyl- $N$-(morpholinomethylene)benzenesulfonamide in

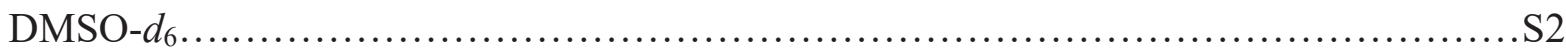

4. Figure S2. ${ }^{13} \mathrm{C}$ NMR spectrum of 4-methyl- $N$-(morpholinomethylene)benzenesulfonamide in

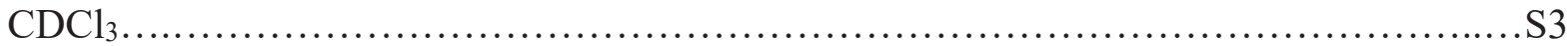

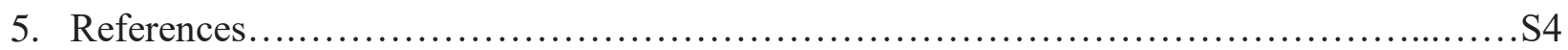




\section{Cycloaddition reaction of sulfonyl azide and phenylacetaldehyde enamine}

We carried out the cycloaddition reaction of sulfonyl azide with phenylacetaldehyde enamine under similar conditions $\left(\mathrm{CDCl}_{3}\right.$, room temperature) as in our earlier published paper for PFAAs. ${ }^{1}$ The amidine product was isolated in $20-40 \%$ yields, accompanied by unanalyzable byproducts. Similar transformation was also reported in the literature. ${ }^{2}$

Below is a specific reaction between tosyl azide and styrylmorpholine (Scheme S1), together with the experimental details and NMR spectra (Figs. S1, S2) of the product.

\section{Synthesis of 4-methyl- $N$-(morpholinomethylene)benzenesulfonamide}

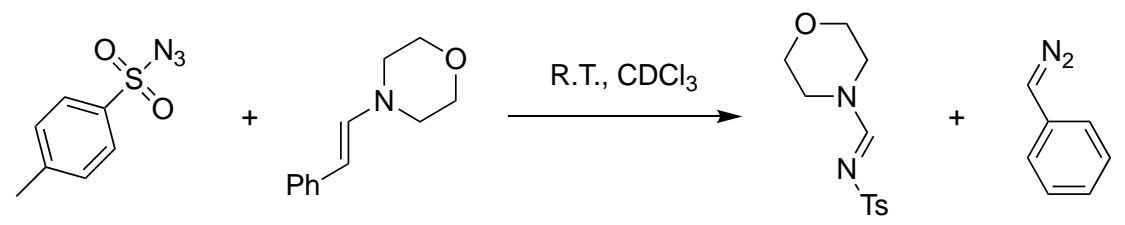

Scheme S1. Reaction of tosyl azide with styrylmorpholine.

To a solution of $(E)$-4-styrylmorpholine $(1.0 \mathrm{mmol})$ in $\mathrm{CDCl}_{3}(1.0 \mathrm{~mL})$, a solution of tosyl azide $(1.1 \mathrm{mmol})$ in $\mathrm{CDCl}_{3}(1.0 \mathrm{~mL})$ was added dropwise while stirring at room temperature. The reaction progress was monitored by NMR spectroscopy. After the completion of the reaction $(\sim 24$ $\mathrm{h}$ ), the solvent was removed under reduced pressure. The crude product was purified by flash column chromatography $($ EtOAc/hexanes $=1: 9)$ to give the amidine product $\left(\mathrm{R}_{\mathrm{f}}=0.2\right)$ as a white powder (yield: 30\%). ${ }^{1} \mathrm{H}$ NMR (500 MHz, DMSO-d $)$ : $\delta_{\mathrm{H}} 2.36\left(\mathrm{~s}, 3 \mathrm{H}, \mathrm{Ar}_{-} \mathrm{CH}_{3}\right), 3.58(\mathrm{~m}, 8 \mathrm{H}$, morpholine $\mathrm{H}), 7.34$ (d, 2H, Ar-H, $\left.\mathrm{J}_{\mathrm{HH}}=8.1 \mathrm{~Hz}\right), 7.65\left(\mathrm{dm}, 1 \mathrm{H}, \mathrm{Ar}-\mathrm{H}, \mathrm{J}_{\mathrm{HH}}=8.2 \mathrm{~Hz}\right), 8.29(\mathrm{~s}, 1 \mathrm{H}$, $\mathrm{NC}-H) ;{ }^{13} \mathrm{C} \mathrm{NMR}\left(125 \mathrm{MHz}, \mathrm{CDCl}_{3}\right): \delta 21.64,44.31,50.41,66.04,66.92,126.69,129.51,139.21$, 142.83, 157.69. 


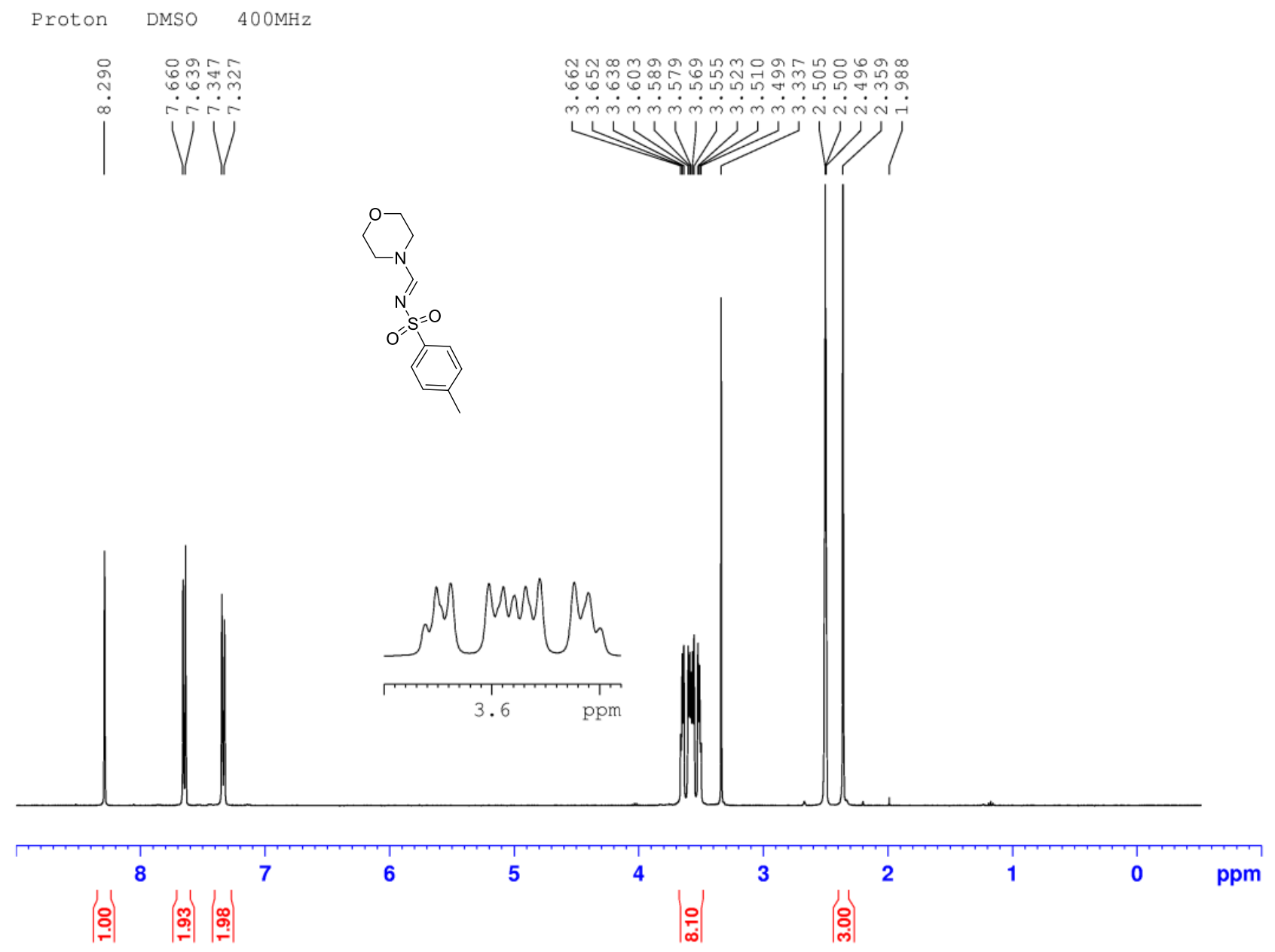

Figure S1. ${ }^{1} \mathrm{H}$ NMR spectrum of 4-methyl- $N$-(morpholinomethylene)benzenesulfonamide in DMSO- $d_{6}$. 


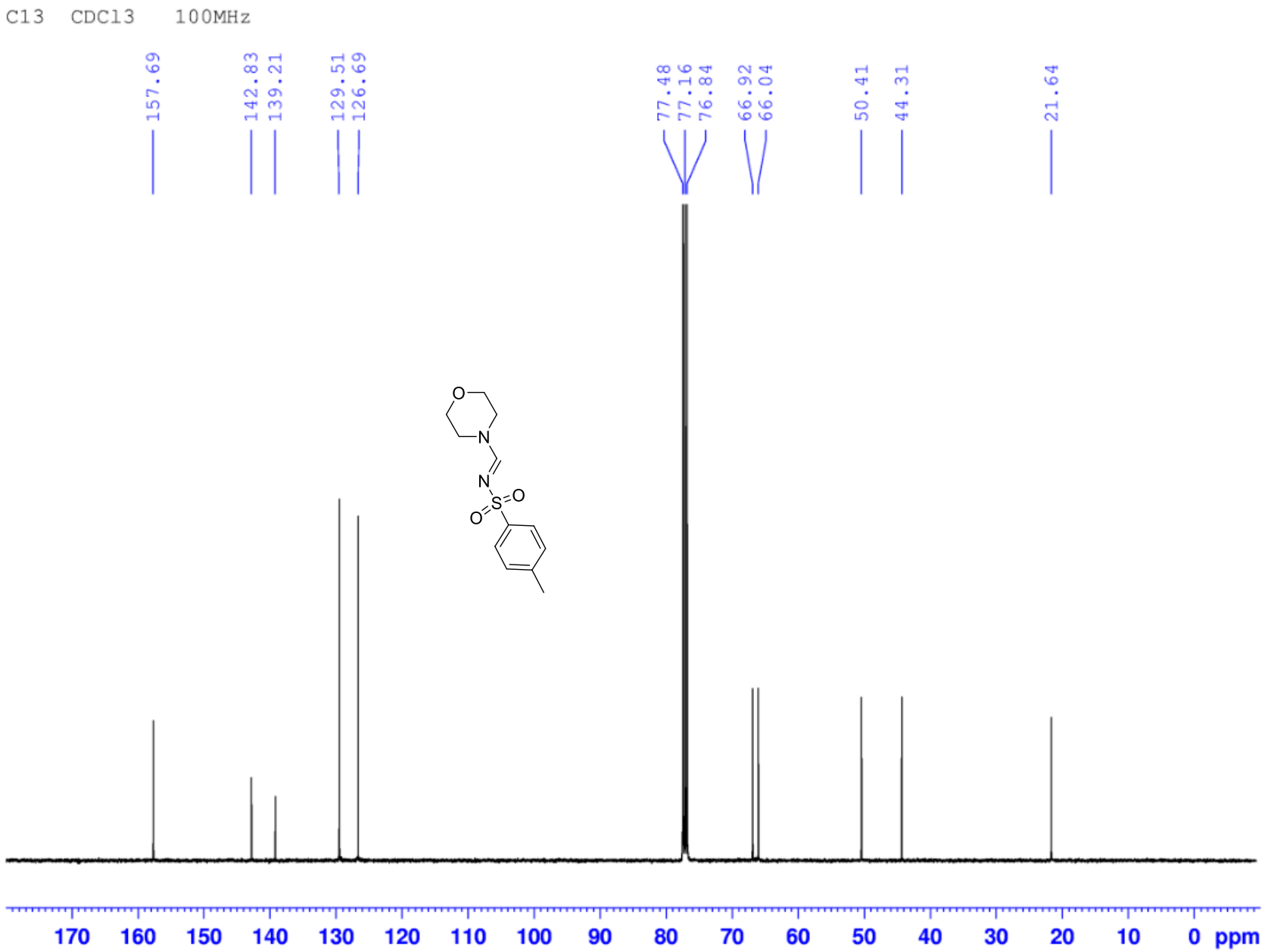

Figure S2. ${ }^{13} \mathrm{C}$ NMR spectrum of 4-methyl- $N$-(morpholinomethylene)benzenesulfonamide in $\mathrm{CDCl}_{3}$. 


\section{References}

1. Xie, S.; Lopez, S. A.; Ramstrom, O.; Yan, M.; Houk, K. N. 1,3-Dipolar cycloaddition reactivities of perfluorinated aryl azides with enamines and strained dipolarophiles. J. Am. Chem. Soc. 2015, 137, 2958-2966.

2. Efimov, I.; Bakulev, V.; Beliaev, N.; Beryozkina, T.; Knippschild, U.; Leban, J.; Zhi-Jin, F.; Eltsov, O.; Slepukhin, P.; Ezhikova, M.; Dehaen, W. Reactions of $\beta$-Azolylenamines with Sulfonyl Azides as an Approach to $N$-Unsubstituted 1,2,3-Triazoles and Ethene-1,2-diamines. Eur. J. Org. Chem. 2014, 3684-3689. 